\title{
Peptidylarginine deiminase inhibition is immunomodulatory and vasculoprotective in murine lupus
}

\author{
Jason S. Knight, ${ }^{1}$ Wenpu Zhao, ${ }^{1}$ Wei Luo, ${ }^{2}$ Venkataraman Subramanian, ${ }^{3}$ Alexander A. O'Dell, ${ }^{1}$ \\ Srilakshmi Yalavarthi, ${ }^{1}$ Jeffrey B. Hodgin, ${ }^{4}$ Daniel T. Eitzman, ${ }^{2}$ \\ Paul R. Thompson, ${ }^{3}$ and Mariana J. Kaplan ${ }^{1}$
}

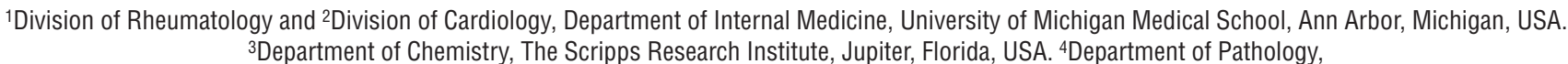
University of Michigan Medical School, Ann Arbor, Michigan, USA.

\begin{abstract}
Recent evidence suggests that enhanced neutrophil extracellular trap (NET) formation activates plasmacytoid dendritic cells and serves as a source of autoantigens in SLE. We propose that aberrant NET formation is also linked to organ damage and to the premature vascular disease characteristic of human SLE. Here, we demonstrate enhanced NET formation in the New Zealand mixed 2328 (NZM) model of murine lupus. NZM mice also developed autoantibodies to NETs as well as the ortholog of human cathelicidin/LL37 (CRAMP), a molecule externalized in the NETs. NZM mice were treated with Cl-amidine, an inhibitor of peptidylarginine deiminases (PAD), to block NET formation and were evaluated for lupus-like disease activity, endothelial function, and prothrombotic phenotype. Cl-amidine treatment inhibited NZM NET formation in vivo and significantly altered circulating autoantibody profiles and complement levels while reducing glomerular IgG deposition. Further, $\mathrm{Cl}$-amidine increased the differentiation capacity of bone marrow endothelial progenitor cells, improved endothelium-dependent vasorelaxation, and markedly delayed time to arterial thrombosis induced by photochemical injury. Overall, these findings suggest that PAD inhibition can modulate phenotypes crucial for lupus pathogenesis and disease activity and may represent an important strategy for mitigating cardiovascular risk in lupus patients.
\end{abstract}

\section{Introduction}

SLE is an autoimmune syndrome of markedly heterogeneous clinical manifestations that preferentially affects women of childbearing age. SLE is characterized by autoantibody formation against nuclear antigens, with resultant immune complex deposition and inflammation in organs such as the kidney, skin, and joints. There is a striking increase in the development of cardiovascular (CV) complications due to accelerated atherosclerotic disease in patients with SLE, which represents an important cause of morbidity and mortality in patients afflicted by this disease $(1,2)$. Type I IFNs have been proposed to be crucial players in the development, progression, and clinical manifestations of SLE as well as in the development of premature CV complications (3-5). While intensive study has shown that both $\mathrm{T}$ and $\mathrm{B}$ cells are required for the lupus phenotype $(6,7)$, neutrophils and other cellular mediators of the innate immune response have, in comparison, received considerably less attention (8).

Neutrophils, the most abundant leukocyte population in peripheral blood, are the first line of defense against microbes, targeting pathogens through a number of mechanisms (9). Included in these is the extrusion of a chromatin meshwork decorated with granular antimicrobial proteins, so-called neutrophil extracellular trap (NET) formation (10-12). At least some patients with SLE have an impaired ability to degrade NETs $(13,14)$, which might explain the long-standing recognition of increased circulating DNA in lupus patients (15). Further, in 2011, several manuscripts reported

Conflict of interest: The authors have declared that no conflict of interest exists. Citation for this article: J Clin Invest. 2013;123(7):2981-2993. doi:10.1172/JCI67390. ex vivo models of enhanced NET formation in SLE patients, while also demonstrating that NETs stimulate plasmacytoid DCs (pDCs) to release type I IFNs such as IFN- $\alpha$ (16-18). NETs may also externalize novel antigens, such as posttranslationally modified histones, that could promote autoantibody formation (19); another example is cathelicidin/LL37, which is exposed in NETs and circulates in complex with both DNA and autoantibodies in lupus patients (16). There are also indications that NETs may be a source of vascular and organ damage in SLE (18). Despite correlative studies linking NETs to human SLE, the association has yet to be definitively addressed in animal models.

At present, there is no gold standard for NET inhibition. One strategy employed in in vitro studies (12) - as well as in vivo modeling of transfusion-related acute lung injury (TRALI) and sepsis $(20-22)$ - is the degradation of NETs with deoxyribonuclease (DNase). But whether DNase treatment is a feasible approach to treating mice over the months it takes to develop a lupus-like phenotype in most strains is unclear $(23,24)$. Of possible genetic approaches, mutations in both NADPH oxidase and peptidylarginine deiminase 4 (PAD4) significantly abrogate NET release without affecting mouse viability (25-27).

Here, we tested whether treatment of the lupus-prone mouse model New Zealand mixed 2328 (NZM) - a model of lupus driven by type I IFNs and characterized by accelerated vascular dysfunction and prothrombotic risk $(28,29)$ - with a chemical inhibitor of PAD enzymes would improve the lupus phenotype and related vascular complications. Indeed, N- $\alpha$-benzoyl-N5-(2-chloro-1-iminoethyl)-Lornithine amide, or $\mathrm{Cl}$-amidine (30), has previously been shown to inhibit PADs in mice without significant toxicity and to improve 

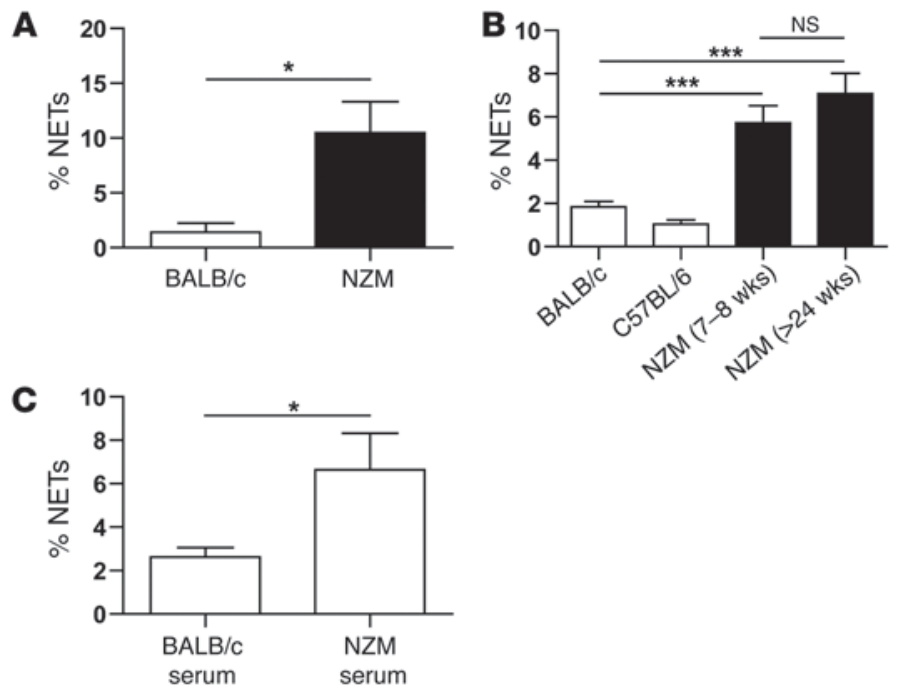

D
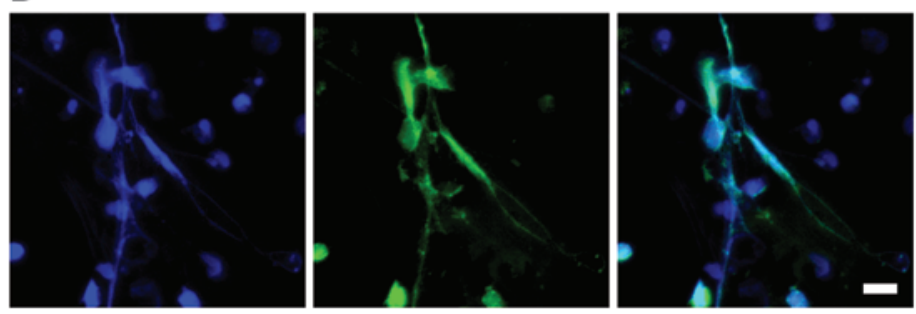

\section{Figure 1}

NZM neutrophils show enhanced release of NETs. Peripheral blood (A) or bone marrow (B) neutrophils from NZM, BALB/C, or C57BL/6 mice were incubated in the absence of serum for 4 hours. Unless otherwise indicated, mice were more than 24 weeks old. (C) BALB/c neutrophils were incubated with $2 \%$ serum from mice older than 24 weeks for 4 hours. In all experiments, NET formation was quantified by fluorescence microscopy as described in Methods. For all experiments, data are shown as mean \pm SEM, and at least 3 independent experiments are represented. ${ }^{\star} P<0.05$; ${ }^{\star * \star} P<0.001$. (D) Representative fluorescence microscopy image showing NET release from NZM neutrophils. DNA is stained blue, and neutrophil elastase is stained green. The right panel overlays the 2 colors. Original magnification, $\times 400$. Scale bar: 10 microns. disease phenotypes in animal models of inflammatory arthritis and inflammatory bowel disease $(31,32)$. PADs are a family of 5 calcium-dependent enzymes (PAD1, -2, -3, -4, and -6) that catalyze the conversion of peptidyl-arginine residues to peptidyl-citrulline by a hydrolytic reaction, which is variably termed deimination or citrullination (33). PAD4 is a histone-modifying enzyme, and inhibition of PAD4 by either genetic knockout or Cl-amidine treatment has been shown to prevent NET formation $(25,26)$.

Here, we first assessed whether NZM mice replicate features of human lupus associated with NET formation. These included measuring the tendency toward enhanced baseline NET formation, the ability of NET-derived material to serve as a source of autoantigens, and the deposition of NET-like material in diseased tissues. We then suppressed NET formation in vivo and studied the extent to which this inhibition ameliorated phenotypes of both lupus activity and vascular damage.

\section{Results}

NZM neutrophils are primed to release NETs. Neutrophils isolated from either peripheral blood (Figure 1A) or bone marrow (Figure 1B) of NZM mice demonstrated significantly enhanced NET formation, as compared with BALB/c and C57BL/ 6 control mice. This was observed in both healthy, prenephritic mice ( 7 to 8 weeks old) and older mice with overt kidney disease (>24 weeks old) (Figure $1 \mathrm{~B}$ ). This phenotype was at least partially dependent on factors present in serum, as treatment of $\mathrm{BALB} / \mathrm{c}$ neutrophils with NZM, but not control, serum resulted in significant increases in NET release (Figure 1C). NZM NETs were reminiscent of human NETs and stained positively for neutrophil elastase, myeloperoxidase (MPO), and citrullinated histone H3 (Figure 1D and
Supplemental Figure 1, A and B; supplemental material available online with this article; doi:10.1172/JCI67390DS1). These results indicate that, similarly to what occurs in human SLE, neutrophils from lupus-prone mice are primed to release NETs and that this phenomenon is induced, at least in part, by factors present in murine lupus serum.

NZM IgG binds to NETs. NET-derived material is a potential source of autoantigens in SLE and, indeed, IgG from NZM serum bound to NETs more strongly than IgG from nonautoimmune mice (Figure 2A). At higher magnification, staining with NZM serum assumed a granular pattern suggestive of reaction with proteins externalized in the NETs (Supplemental Figure 1, C and D). To follow a more quantitative approach, we developed ELISAs to both stimulated neutrophil supernatants (which contained NETs) and to CRAMP, the ortholog of human cathelicidin/LL37. Indeed, serum from older NZM mice ( $>24$ weeks old) contained autoantibodies to both NETs in general and to CRAMP in particular (Figure 2B). Notably, those mice that had the highest reactivity to NETs also had high reactivity to CRAMP (data not shown). To further confirm that NZM serum reacts with the protein fraction of NETs and not simply DNA, unstimulated neutrophil lysates, or NETs, were resolved by SDS-PAGE and probed with NZM serum. NZM, but not control, IgG showed strong reactivity with several neutrophil proteins, an effect that was enhanced when NETs were compared with unstimulated neutrophil lysates (Figure 2C). These results indicate that lupus-prone mice develop autoantibodies to NET components, including NET proteins.

Lupus NETs induce endothelial dysfunction and infiltrate tissues. Our group has previously reported NET-like material in the kidneys and skin of lupus patients with active nephritis and cutaneous 
A
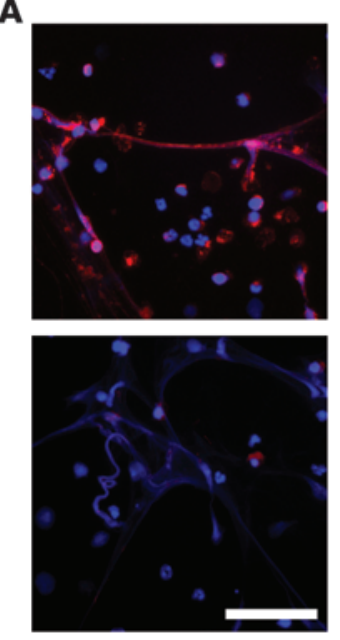

B
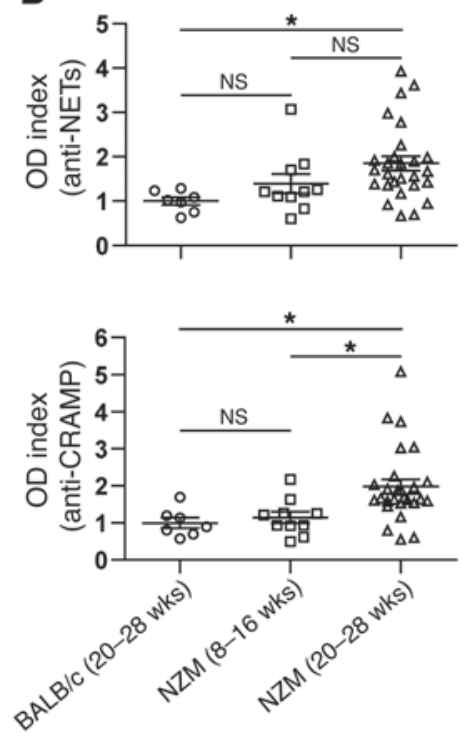

\section{Figure 2}

NZM IgG binds to NETs and to proteins derived from NETs. (A) Paraformaldehyde-fixed NZM NETs were incubated with $1 \%$ serum from NZM (top panel) or BALB/c (bottom panel) mice more than 24 weeks old. Bound IgG was detected with Texas red-conjugated anti-mouse IgG. DNA is stained blue with Hoechst 33342. Original magnification, $\times 400$. Scale bar: 50 microns. (B) Serum was tested for autoantibodies to NETs or CRAMP as described in Methods. OD index compares absorbance to the mean value for BALB/c controls. Each data point represents an individual mouse, with mean and SEM shown for each group. ${ }^{*} P<0.05$. (C) NETs were generated as described in Methods. $20 \mu \mathrm{g}$ of either NET protein or unstimulated neutrophil lysate (Lys) was resolved by $15 \%$ SDS-PAGE and probed with $0.5 \% \mathrm{NZM}$ or BALB/c serum; bands were detected with HRP-conjugated anti-mouse IgG. Blots were also probed with anti-MPO to ensure equal loading. Data in $\mathbf{A}$ and $\mathbf{C}$ are representative of at least 3 independent experiments.

lupus, respectively (18). This is reminiscent of similar deposition reported in patients with antineutrophil cytoplasmic antibodyassociated (ANCA-associated) vasculitis (34) as well as in a mouse model with elements of both ANCA-associated vasculitis and lupus (35). Here, we detected deposits of MPO colocalizing with DNA in the kidneys of older ( $>24$ weeks old) NZM mice (Supplemental Figure 2A), while these were not observed in either 8-weekold NZM mice or nonautoimmune controls (data not shown).

We have also previously shown that netting neutrophils from SLE patients can damage the endothelium (18) and that NZM miceand related murine models - develop impaired endothelial function as they age $(28,36)$. Here, we found that preincubation of mouse aortic rings from 8-week-old NZM mice with NETs resulted in significantly impaired vasorelaxation in response to acetylcholine (Ach) (Supplemental Figure 2B). These results indicate that NETs can directly induce endothelial dysfunction in lupus-prone mice.

The PAD inbibitor Cl-amidine blocks NET release from NZM neutrophils in vitro. We first asked whether NET release from NZM neutrophils could be blocked by a PAD inhibitor in vitro. Histone hypercitrullination by PAD4 has previously been shown to be critical for histone decondensation and NET release $(25,26)$. Cl-amidine, which covalently modifies a PAD active-site cysteine, resulting in irreversible inactivation of the enzyme, is a highly specific inhibitor of PAD4 and related intracellular and extracellular PAD isozymes $(30,37)$. Indeed, Cl-amidine prevented hypercitrullination of histone H3 in NZM neutrophils, as shown by Western blot in

C

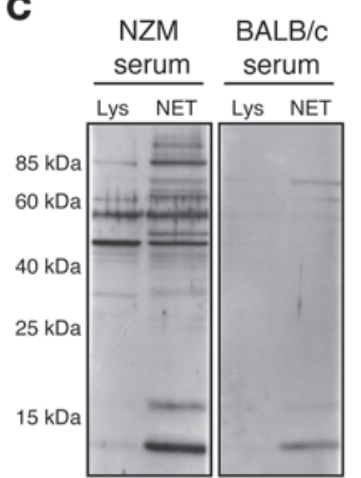

Anti-MPO

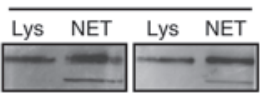

Figure 3A, and blocked NET release triggered by PMA stimulation (Figure $3 \mathrm{~B})$. Cl-amidine also prevented NZM serum-triggered NET release from BALB/c neutrophils (Figure 3C). The effect was specific for NET formation, as Cl-amidine did not inhibit the PMA-triggered oxidative burst as measured by $\mathrm{H}_{2} \mathrm{O}_{2}$ release (Figure 3D). These results indicate that $\mathrm{Cl}$-amidine effectively inhibits NET formation by murine lupus neutrophils.

Previous studies have shown that daily Cl-amidine treatment does not alter the frequency or absolute number of T cells (total and subsets), B cells, NK cells, or monocytes (32). We therefore predicted that $\mathrm{Cl}$-amidine, in contrast to what we observed for neutrophils and NET formation, would not robustly inhibit lymphocyte phenotype and function in vitro. Indeed, Cl-amidine did not affect cellsurface upregulation of the activation markers CD25 and CD69 on T cells in response to anti-CD3/CD28 stimulation (Figure 3E, Supplemental Figure $4 \mathrm{~A}$, and data not shown). Cl-amidine also had no effect on $T$ cell proliferation in response to anti-CD3/CD28 stimulation or B cell proliferation following LPS stimulation over 48 to 72 hours (Figure 3F and Supplemental Figure 4B). Finally, Cl-amidine did not affect lymphocyte apoptosis, as assessed by annexin $\mathrm{V}$ staining (data not shown).

Having confirmed Cl-amidine's relative specificity for neutrophils, we further tested the concept that Cl-amidine can block NET release and thereby prevent end-organ damage by asking whether neutrophils treated with $\mathrm{Cl}$-amidine were less prone to inducing endothelial dysfunction. NZM neutrophils were stimulated to release NETs with PMA, either in the presence or absence of $\mathrm{Cl}$-amidine. Aortic rings from 8-week-old NZM mice were then incubated with neutrophil supernatants, and endothelium-dependent vasorelaxation was assessed by Ach titration. Supernatants from Cl-amidine-treated neutrophils contained fewer NETs and caused less endothelial damage when compared with the control supernatants (Supplemental Figure 3). In contrast, direct treatment of aortic rings with Cl-amidine had no significant effect on vasorelaxation (data not shown). These results indicate that inhibition of NET formation by Cl-amidine can hamper the deleterious effect of lupus neutrophils on the endothelium.

Cl-amidine blocks NET release when delivered by subcutaneous injection. Two groups of 10 female NZM mice were treated with Cl-amidine $(10 \mathrm{mg} / \mathrm{kg} / \mathrm{d})$ or vehicle control by daily subcutaneous injection beginning at 12 weeks of age and continuing through sacrifice at 26 weeks of age; these groups were utilized for the experiments depicted in Figures 4, 5, and 6. From these mice, bone marrow neutrophils were harvested at 26 weeks of age and were incubated either without stimulation or with PMA. Under either condition, neutrophils 
A

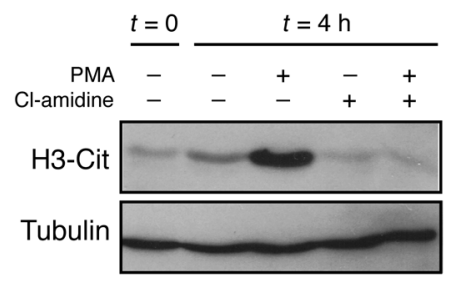

D

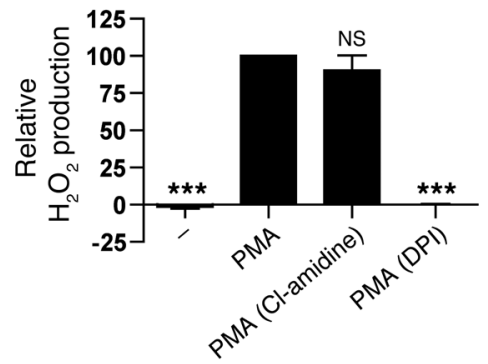

B

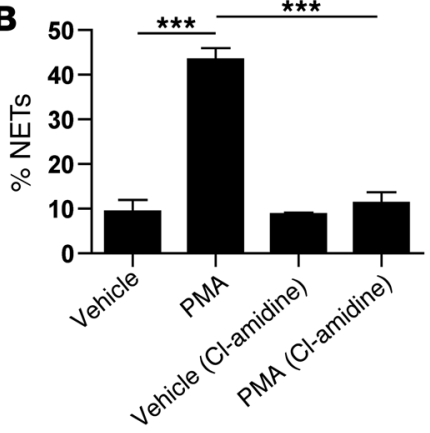

E

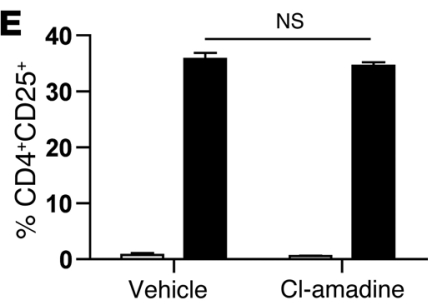

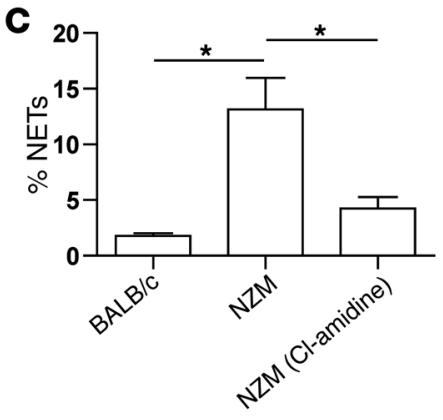

$\mathbf{F}$

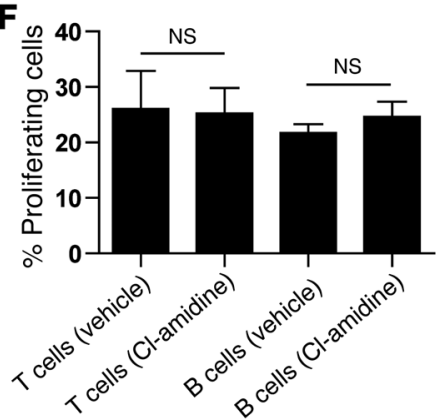

Figure 3

PAD inhibition blocks NZM NET formation in vitro. (A) Cl-amidine treatment inhibits histone H3 citrullination. NZM bone marrow neutrophils were treated with $100 \mathrm{nM}$ PMA in the presence or absence of $200 \mu \mathrm{M} \mathrm{Cl}$-amidine. Whole-cell lysates were resolved by SDS-PAGE. A Western blot representative of 3 independent experiments is shown. H3-Cit, citrullinated histone H3. (B) Cl-amidine treatment inhibits NET formation. NZM neutrophils were treated with PMA in the presence or absence of $\mathrm{Cl}$-amidine for 12 hours. NET formation was determined by fluorescence microscopy. (C) BALB/c neutrophils were incubated with either $2 \%$ BALB/c or NZM serum from mice older than 24 weeks for 12 hours in the presence or absence of $\mathrm{Cl}$-amidine, as indicated in parentheses. (D) $\mathrm{Cl}$-amidine treatment does not alter $\mathrm{H}_{2} \mathrm{O}_{2}$ production. NZM neutrophils were stimulated for 1 hour with PMA in the presence of inhibitors as indicated. The PMA-stimulated sample was arbitrarily set at $100 \% \mathrm{H}_{2} \mathrm{O}_{2}$ production. DPI, NADPH oxidase inhibitor. (E) Cl-amidine treatment does not alter T cell activation. T cells were purified from NZM spleens and stimulated with anti-CD3/CD28 (black bars) in the presence or absence of $\mathrm{Cl}$-amidine. CD25 expression was negligible (white bars) without stimulation. (F) $\mathrm{Cl}$-amidine treatment does not alter lymphocyte proliferation. CFSE-labeled T or B cells were purified from NZM spleens and stimulated with antiCD3/CD28 or LPS, respectively. After 48-72 hours, in the presence or absence of Cl-amidine, proliferating cells were quantified as described in Methods. For all experiments, data are shown as mean \pm SEM, and at least 3 independent experiments are represented. ${ }^{\star} P<0.05$; ${ }^{\star \star \star} P<0.001$.

from the Cl-amidine-treated mice produced significantly fewer NETs (Figure 4A). As mentioned above, serum from NZM, but not control, mice induced BALB/c neutrophils to undergo NET formation; when comparing serum from vehicle- and $\mathrm{Cl}$-amidine-treated NZM mice, only the vehicle-treated serum significantly triggered NET formation in BALB/c neutrophils (Supplemental Figure 5A). Overall, these results show that daily administration of Cl-amidine inhibits NET formation in lupus-prone mice.

Above, we showed that $\mathrm{Cl}$-amidine does not affect $\mathrm{T}$ cell proliferation or activation in vitro. To further address $\mathrm{Cl}$-amidine's specificity for neutrophils in our treatment groups, we quantified serum levels of a number of cytokines relevant to lymphocyte function, including IFN- $\gamma$, IL-2, IL-4, IL-5, and TNF- $\alpha$. Three cytokines (IFN- $\gamma$, IL-2, IL-4) were below the detection limit of the multiplex assay, while levels of serum IL-5 and TNF- $\alpha$ were detected. There were no significant differences in the levels of these serum cytokines between the vehicle- and $\mathrm{Cl}$-amidine-treated groups (Supplemental Figure 5, B and C); there was a significant increase in TNF- $\alpha$ as mice got older, which was independent of $\mathrm{Cl}$-amidine treatment (Supplemental Figure 5B).

Cl-amidine suppresses complement consumption, while altering autoantibody profiles in NZM mice. Complement C3 was recently shown to deposit on NETs, and importantly, patients with impaired NET degradation displayed lower levels of complement C3 and C4 in blood (14); further, NETs can activate the complement cascade in vitro, resulting in deposition of C1q, which seems to further protect NETs from degradation by DNase (14). Clinically, serum C3 levels fall with increasing lupus activity due to accelerated consumption and immune complex formation, especially in the context of lupus nephritis $(38,39)$. As expected, at both 20 and 26 weeks of age, vehicle-treated NZM mice showed a statistically significant decrease in serum C3 levels when compared with 12 -week-old prenephritic NZM mice (Figure 4B). In contrast, the development of hypocomplementemia was hampered in the $\mathrm{Cl}$-amidine-treated mice, in which there was no significant decrease in $\mathrm{C} 3$ levels with increasing age (Figure 4B). When 20- and 26-week-old Cl-amidine-treated mice were directly compared with matched vehicle-treated mice, there was a trend toward higher C3 levels with Cl-amidine treatment, although this did not reach statistical significance. Overall, these results hint that $\mathrm{Cl}$-amidine treatment can abrogate complement consumption characteristic of lupus prone-mice.

Anti-dsDNA autoantibody levels fluctuate in human lupus patients, where they may herald disease flares, and it has previously 
A

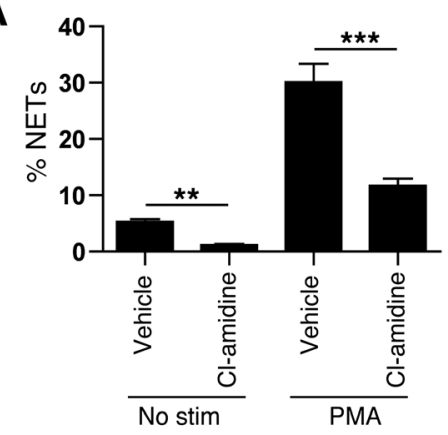

C

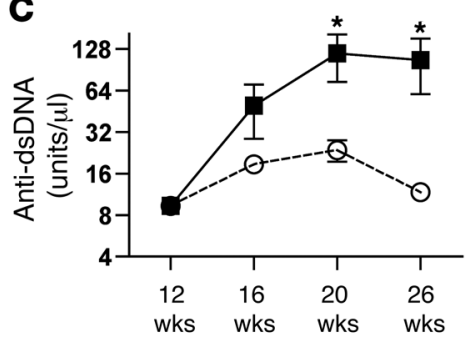

B

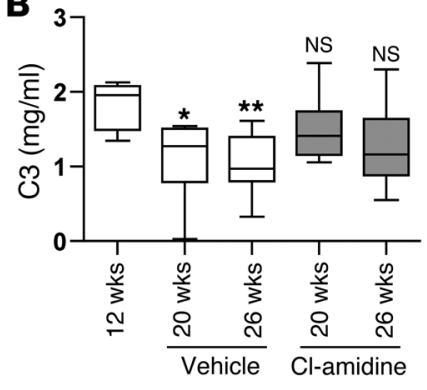

D

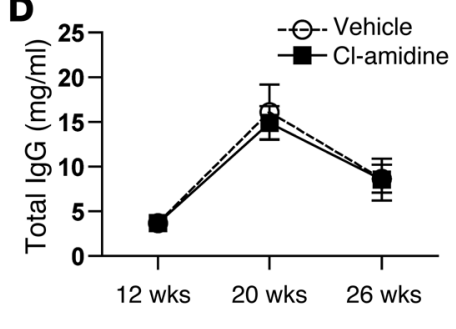

\section{Figure 4}

In vivo, $\mathrm{Cl}$-amidine inhibits NET formation while altering complement levels and the autoantibody profile of NZM mice. Female NZM mice were treated by daily subcutaneous injection with either $\mathrm{Cl}$-amidine $(10 \mathrm{mg} / \mathrm{kg} / \mathrm{d})$ or vehicle control beginning at 12 weeks of age (10 mice per group). Mice were sacrificed at 26 weeks of age. (A) Bone marrow neutrophils from sacrificed mice were incubated either in the absence of serum for 4 hours (no stim) or with 100 nM PMA for 12 hours. NET formation was quantified by fluorescence microscopy. (B-D) Serum from the aforementioned 2 groups was assayed for complement C3 (B), anti-dsDNA antibodies (C), or total IgG (D) using commercial kits. For C3, $P$ values result from comparison to the baseline 12-week group. For anti-dsDNA, $P$ values compare the vehicle and $\mathrm{Cl}$-amidine groups directly. ${ }^{\star} P<0.05 ;{ }^{\star \star} P<0.01 ;{ }^{\star \star \star} P<0.001$. For $\mathbf{A}, \mathbf{C}$, and $\mathbf{D}$, data were plotted as the mean \pm SEM. For $\mathbf{B}$, boxes represent the median, 25th percentile, and 75th percentile, while whiskers delineate the minimum and maximum values. been reported that these antibodies are elevated in NZM mice (40). Indeed, vehicle-treated mice developed an approximately 3 -fold increase in anti-dsDNA levels from 12 weeks to 20 weeks of age, with levels then falling from 20 weeks to 26 weeks, as immune complex deposition accelerated and proteinuria developed. In contrast, Cl-amidine-treated mice showed a different pattern, with a 12 -fold increase in anti-dsDNA levels between 12 and 20 weeks of age and then relatively stable levels thereafter (Figure 4C). While total IgG levels did not differ between the 2 groups (Figure 4D), both anti-NETs and anti-CRAMP autoantibodies mirrored anti-dsDNA and showed a trend toward increased levels in the Clamidine-treated mice (Supplemental Figure 6).

Similar to what is found in human SLE, NZM mice have been reported to express an enhanced type I IFN signature in various compartments and to depend on type I IFN responses for the development of disease manifestations, autoantibody synthesis, and vascular damage $(28,29)$. Given evidence that NETs are a potential stimulus for type I IFN synthesis by pDCs in human lupus (16-18), we investigated whether NET blockade by PAD inhibition could suppress the in vivo type I IFN signature of NZM mice. Although there was a tendency toward suppression of various type I IFNresponsive genes with $\mathrm{Cl}$-amidine treatment (Supplemental Figure $7 \mathrm{~A})$, the trend in the spleen compartment did not reach statistical significance for any of the tested genes at the 26-week time point.

$\mathrm{Cl}$-amidine reduces $M P O, I g G$, and $C 3$ deposition in NZM kidneys. At 26 weeks of age, there was a trend toward less albuminuria in the Cl-amidine-treated mice, although this did not reach statistical significance (Figure 5A). Kidneys were also scored by histopathology as described in Methods, using both activity and chronicity indices that approximate the scoring system of human lupus nephritis; neither index showed a significant difference between the 2 groups (Figure 5B and Supplemental Figure 7B) at the 26-week time point.

One possible explanation for the aforementioned higher circulating $\mathrm{C} 3$ and autoantibody levels in $\mathrm{Cl}$-amidine-treated mice is that $\mathrm{Cl}$-amidine prevents formation and deposition of immune complexes in target organs. Indeed, some studies have demonstrated a drop in anti-dsDNA levels with increasing disease activity $(15,41)$, while there is evidence that increases in extracellular DNA can facilitate anti-dsDNA clearance $(42,43)$. Similar to what is shown in Supplemental Figure 2A, we stained glomeruli for MPO and DNA and quantified discrete areas of MPO/DNA overlap as a measure of neutrophil and NET infiltration. Indeed, mice treated with Clamidine had less MPO in their kidneys than controls (Figure 5C).

Further, glomerular IgG and C3 were quantified by immunofluorescence microscopy. Similar to the MPO data, there was significantly less IgG deposition and a strong trend toward less C3 deposition in $\mathrm{Cl}$-amidine-treated mice as compared with controls (Figure 5D). Importantly, there was also a significant correlation between IgG and C3 deposition (Supplemental Figure 7C) as well as an inverse correlation between IgG deposition and serum antidsDNA levels (Supplemental Figure 7D). Overall, this data suggests that inhibition of NET formation can limit the deposition of C3, anti-dsDNA, and immune complexes in target tissues, which may then result in higher circulating levels of these disease markers, while diminishing tissue damage.

Cl-amidine improves vasculogenesis and endothelium-dependent vasorelaxation in vivo in NZM mice. Previous work from our group indicates that, similar to patients affected by SLE, NZM mice and similar lupus models have impairments in the phenotype and function of cells involved in vascular repair following endothelial cell injury: the bone marrow-derived endothelial progenitor cells (EPCs). This phenotype has been linked to both type I IFNs and to the presence of aberrant neutrophils $(28,36,44,45)$. Indeed, treatment with Cl-amidine from 12 weeks of age through 26 weeks of age significantly improved the capacity of bone marrow EPCs to differentiate into mature endothelial cells (Figure 6A).

We also tested whether in vivo exposure to Cl-amidine would improve endothelium-dependent vasorelaxation. Using the same groups of mice described above, we found that treatment with Cl-amidine significantly improved endothelium-dependent vasorelaxation of aortas harvested at euthanasia (Figure 6B). Importantly, the in vitro introduction of $\mathrm{Cl}$-amidine alone did not have an effect on either the endothelial cell differentiation or aortic 
A

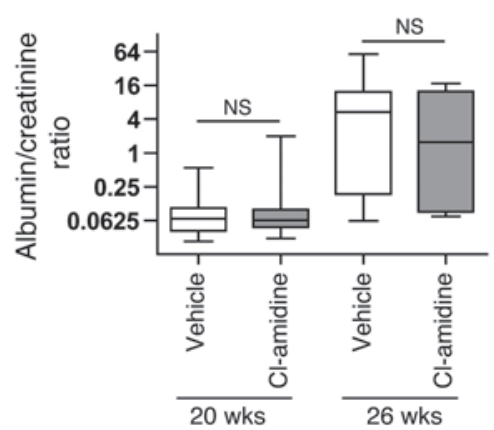

C
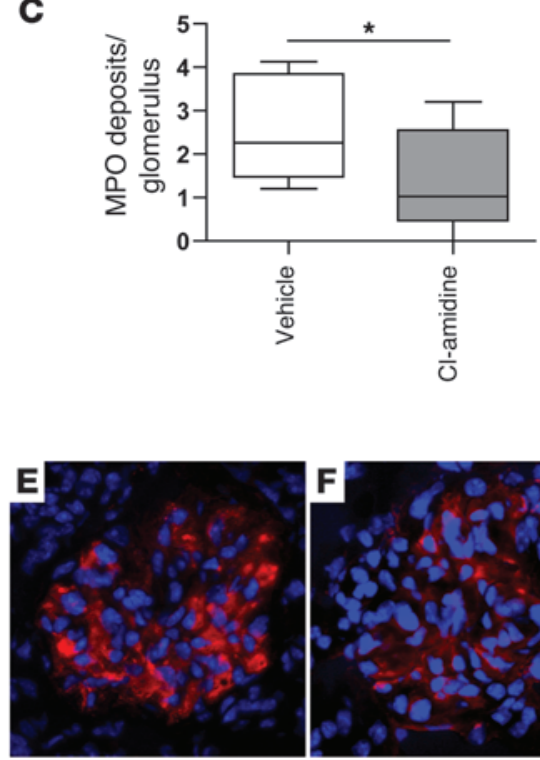

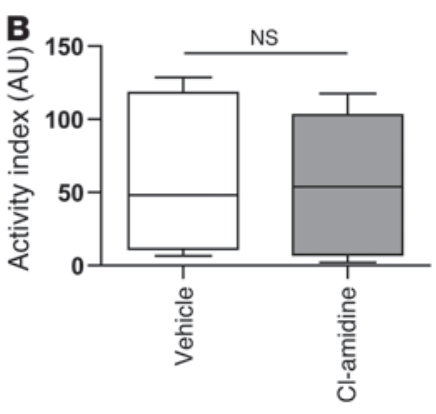

D
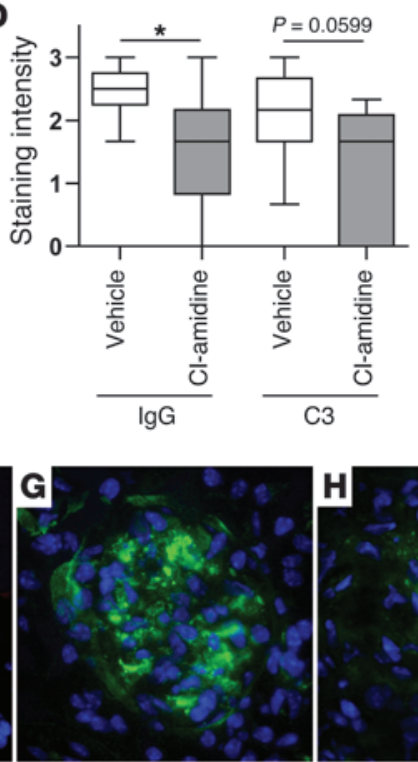

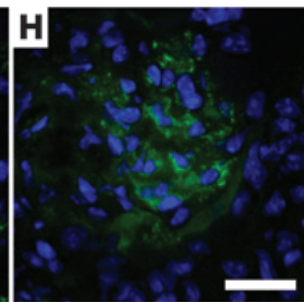

\section{Figure 5}

PAD inhibition reduces MPO and immune complex deposition in the kidneys of NZM mice. (A) Urine albumin/ creatinine ratios were determined for the 2 groups of NZM mice presented in Figure 4. (B) 3-micron sections were prepared from formalin-fixed kidneys of 26-week-old mice, and activity index was calculated as described in Methods. (C) Glomerular MPO deposition was determined as described in Methods and in Supplemental Figure 2. Discrete areas of MPO staining were counted, with at least 10 glomeruli considered per mouse. (D) Glomeruli were also stained for IgG and C3 deposition. At least 10 glomeruli were scored for staining intensity on a scale of 0 to $3+$, with average intensity then calculated for each kidney. ${ }^{\star} P<0.05$. One $P$ value that approaches significance is presented as a number. Box-and-whisker plots represent 10 mice per group, with boxes representing the median, 25th percentile, and 75th percentile, while whiskers delineate the minimum and maximum values. (E-H) Representative glomeruli from the vehicle group ( $\mathbf{E}$ and $\mathbf{G}$ ) and the $\mathrm{Cl}$-amidine group ( $\mathbf{F}$ and $\mathbf{H})$. IgG is stained red and $\mathrm{C} 3$ is stained green. Original magnification, $\times 400$. Scale bar: 25 microns. relaxation assays (data not shown). These results indicate that Cl-amidine significantly improves vasculogenesis and endothelial function in lupus-prone mice.

Both DNase and Cl-amidine delay thrombosis development in NZM mice. NETs predispose to deep vein thrombosis (DVT) in baboons, mice, and humans, where they bind platelets and represent an integral part of the thrombus scaffolding (46-48). In addition, serine proteases derived from NETs activate the coagulation cascade by degrading tissue factor pathway inhibitor (49) while also promoting the activation of factor XII (50). Lupus patients are prone to thrombosis, even in the absence of anti-phospholipid antibodies (51), and previous work from our group has demonstrated that NZM lupus-prone mice exhibit rapid arterial thrombosis in response to endothelial injury, when compared with nonautoimmune control mice (28). Given the association between other hyper- coagulable disease states - such as cancer - and NETs (52), we hypothesized that NETs contribute to the accelerated thrombosis previously reported in NZM mice. Specifically, we tested whether inhibition of NETs would mitigate arterial thrombosis in response to photochemical injury of the common carotid artery (28).

For these studies, 20-week-old prenephritic NZM mice were treated with either $\mathrm{Cl}$-amidine or vehicle by daily subcutaneous injection for 1 week. Importantly, this 1 -week course of treatment was sufficient to significantly inhibit NET formation in bone marrow neutrophils (Supplemental Figure 8). In addition to Cl-amidine treatment, we also studied a group of mice in which DNase was administered in vivo to disrupt NETs, as previously described $(47,52)$.

DNase treatment modified the content of carotid artery thrombi, as determined by immunohistochemistry (Figure 7A). Citrullinated histone $\mathrm{H} 3$ and CRAMP have both been used as surrogates

\section{Figure 6}

PAD inhibition improves endothelial cell differentiation and endothelium-dependent vasorelaxation in NZM mice. (A) For the 2 groups of mice presented in Figure 4, EPCs were identified as bone marrow cells capable of differentiating into mature endothelial cells (defined by coexpression of acetylated LDL and Lectin I as described in Methods). (B) Aortic rings were isolated from the same 2 groups of mice, and Ach-dependent relaxation was determined as described in Methods. Data are presented as the mean \pm SEM for 10 mice per group. ${ }^{\star \star} P<0.01 ;{ }^{* \star *} P<0.001$.
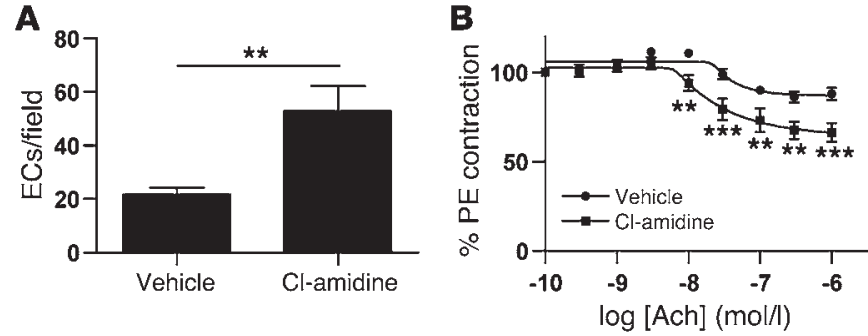
A
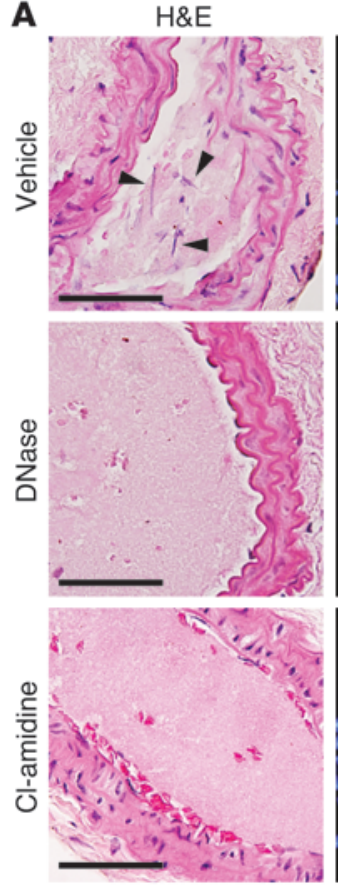

D
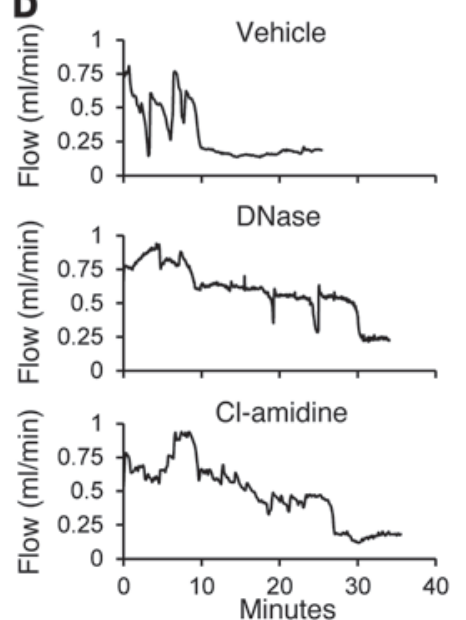

Hoechst
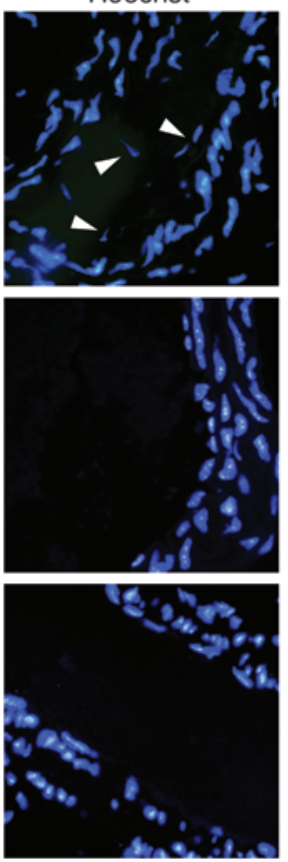

E
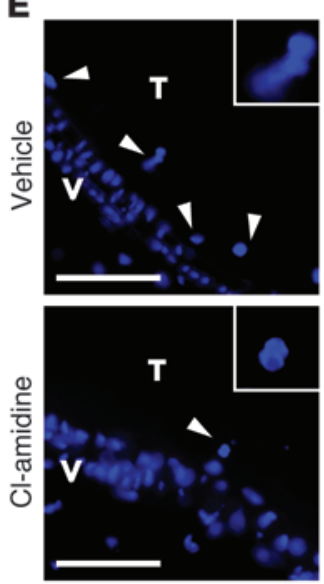

H3-Cit
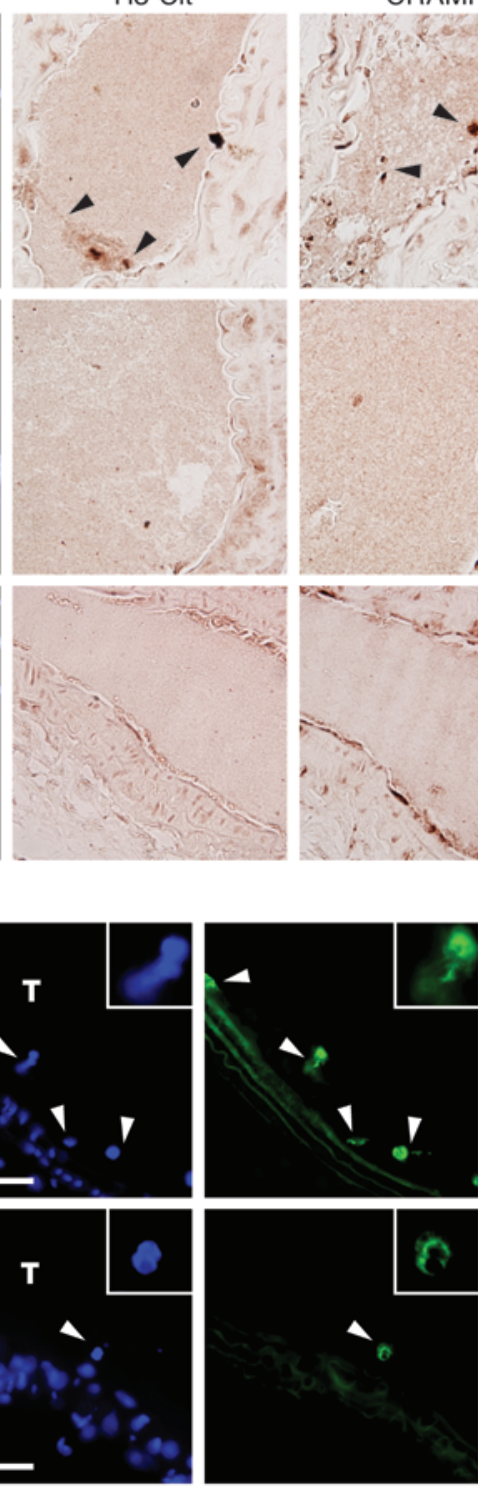

CRAMP
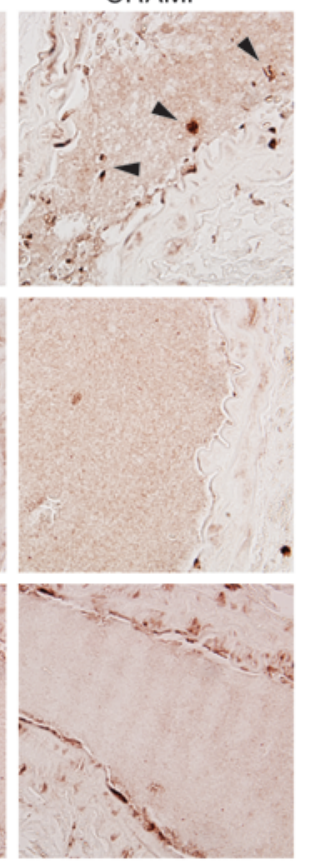

B

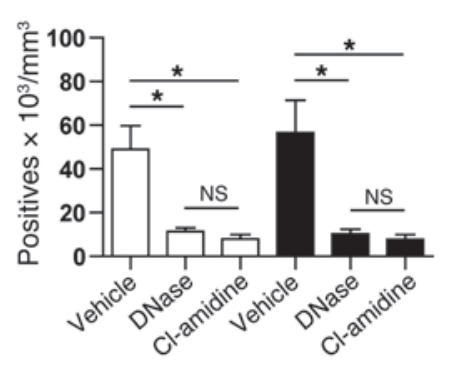

C

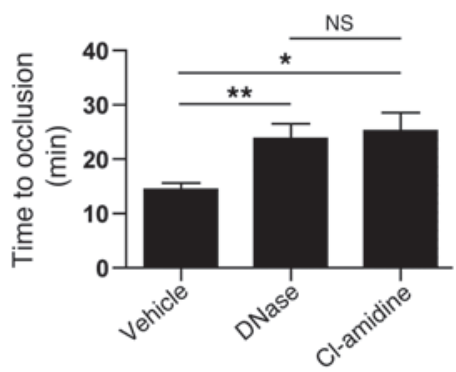

Figure 7

PAD inhibition prolongs time to arterial thrombosis and reduces NET density in NZM mice. 20-week-old NZM mice were treated with Cl-amidine $(10 \mathrm{mg} / \mathrm{kg} / \mathrm{d})$ or vehicle, by daily subcutaneous injection for 1 week. Carotid artery thrombosis was then induced by photochemical injury, with DNase administered just prior to injury in some vehicle-treated mice. (A) Both DNase and $\mathrm{Cl}$-amidine change the content of thrombi, resulting in the capture of fewer citrullinated histone H3-positive (H3-Cit) and CRAMP-positive structures. H\&E and Hoechst 33342 (DNA in blue) staining are also shown for these representative paraffin-embedded sections. Arrowheads highlight NET-like structures. (B) For each thrombus, at least 3 sections were quantified for H3-Cit-positive (white bars) and CRAMP-positive (black bars) structures ( $n=5$ mice per group). (C and D) Both DNase and $\mathrm{Cl}$-amidine prolong time to vessel occlusion ( $n=10$ for vehicle and $\mathrm{Cl}$-amidine groups; $n=5$ for the DNase group), with representative carotid artery flow tracings in D. (E) Cl-amidine reduces the NET density of carotid thrombi. Frozen sections were stained with Hoechst 33342 (DNA in blue) and anti-CRAMP (green). Representative images are shown, with overlays to the right. V, vessel wall; T, thrombus. (F) For each thrombus, at least 3 sections were quantified for discrete areas of DNA/CRAMP overlap ( $n=5$ per group). Original magnification, $\times 1000$. Scale bars: 50 microns. Volumes were determined by multiplying thrombus area by section depth. All quantification is presented as mean \pm SEM. ${ }^{*} P<0.05$; ${ }^{* *} P<0.01$.

for neutrophils undergoing NET formation in vascular tissue (47, $50,53,54)$, and indeed, cells and cell remnants staining positive for either marker were less likely to be captured in the thrombi of DNase-treated mice (Figure 7B). Further, not only did DNase treatment change the content of the thrombi, but time to vessel occlusion was also significantly delayed by DNase (quantified in
Figure 7C, with representative flow tracings in Figure 7D). Taken together, these data quantitatively support the importance of NET formation in this arterial thrombosis model in NZM mice.

Similar to DNase, Cl-amidine treatment modified the content of carotid artery thrombi, with quantitatively less citrullinated histone H3- and CRAMP-positive material identified in the throm- 
bi (Figure 7, A and B). Further, and again similar to DNase, time to vessel occlusion was significantly prolonged by $\mathrm{Cl}$-amidine treatment (quantified in Figure 7C, with representative flow tracings in Figure 7D). To more definitively prove that the CRAMP-positive material represents NETs, a portion of vehicle- and Cl-amidinetreated thrombi were analyzed by immunofluorescence. Indeed, overlapping staining for DNA and CRAMP, strongly reminiscent of NETs, was easily detected in the vehicle-treated thrombi, but only rarely in $\mathrm{Cl}$-amidine-treated counterparts (representative staining in Figure 7E with quantification in Figure 7F). Further, when rare CRAMP-positive cells were detected in Cl-amidinetreated thrombi, they had the appearance of intact neutrophils, without mixing of nuclear DNA and cytoplasmic CRAMP signals (Figure 7E). Overall, these results show that inhibition of NET formation by $\mathrm{Cl}$-amidine or disruption of formed NETs by DNase significantly mitigates the prothrombotic phenotype of NZM mice.

\section{Discussion}

In human SLE, we have previously shown that aberrant neutrophils promote endothelial damage and abnormal endothelial differentiation, and we have posited that these cells play a critical role in the well-recognized accelerated atherosclerosis of SLE patients $(44,55)$. Furthermore, in experimental systems, depletion of neutrophils can protect against antibody-mediated glomerulonephritis (56) and lupus serum from human patients can induce neutrophil-mediated organ damage (57). The presence of neutrophilic infiltrates has been recognized as an early feature of glomerulonephritis $(58,59)$, while proteins released from neutrophilic granules are toxic to glomerular structures (59-61) and circulate at elevated levels in the blood (62-64). To these previous observations, a role has recently been hypothesized for NET formation in the pathogenesis of - and organ damage associated with - SLE (16-18).

Given the hypothesized role for NETs as inducers of type I IFNs, we favored the NZM model of murine lupus for the experiments described here (40). The NZM lupus phenotype - similar to the related strain New Zealand black/New Zealand white $F_{1}(65)-$ is strongly dependent on the presence of the type I IFN receptor $(28,29)$. This is in contrast with other models of murine lupus, such as MRL/lpr, where deletion of the type I IFN receptor actually results in more severe disease (66); similarly, in MRL/lpr mice, genetic deletion of the Nox2 gene - which disrupts NADPH oxidase function and at least 1 of the pathways to NET formation $(25,26,67)-$ results in a more severe lupus phenotype (68). Our study therefore differs in at least 2 important ways from the Nox2deficient MRL/lpr model (68), in that we are inhibiting NET formation in a type I IFN-dependent model and Cl-amidine does not interfere with ROS formation (which may play a protective role in lupus). Indeed, data to date points toward ROS formation being upstream of PAD4 activation in neutrophils (69), supporting the idea that PAD inhibition is a more targeted strategy for inhibiting NET formation. Given the ability of $\mathrm{Cl}$-amidine to also improve other inflammatory disease phenotypes $(31,32)$, it will be of interest to revisit those inflammatory murine models in the context of what we now know about Cl-amidine's effectiveness in inhibiting NET formation in mice.

Here, we have shown that NZM mice replicate a number of the features of aberrant NET formation in human lupus. Paralleling work by Lande et al. in SLE patients (16), NZM neutrophils exhibit higher spontaneous NET formation than controls when studied ex vivo, and NZM mice develop autoantibodies to the murine ortholog of cathelicidin/LL37, CRAMP. Further, similar to the characterization of SLE serum by Garcia-Romo et al. (17), NZM serum stimulates control neutrophils to release NETs. The type I IFN dependence of this serum phenomenon in NZM mice remains unclear, although NZM serum-induced NET formation was still observed in type I IFN receptor-knockout neutrophils (data not shown), suggesting that additional factors - potentially including other cytokines and autoantibodies - must play a role.

Reminiscent of human data reported by our group (18), we detected deposits of NET-like material in NZM kidneys. We were also able to detect rare netting neutrophils in nonexposed, unaffected NZM skin (data not shown), although we are unable to comment on their association with cutaneous lupus, as these mice do not develop typical spontaneous lesions. The recently described tape stripping model in New Zealand black/New Zealand white $F_{1}$ mice is, however, a place where the importance of NET formation in skin disease could eventually be experimentally tested (70). Another feature of human lupus is that some patients do not degrade NETs normally $(13,14)$. Future studies should address whether a similar phenotype is observed in murine lupus strains.

The PAD inhibitor Cl-amidine robustly inhibited NET formation in vitro, as has been demonstrated by others (25), while daily subcutaneous treatment with the drug inhibited NET formation when neutrophils were studied ex vivo. As there is no standardized biomarker for in vivo NET formation, it is difficult to prove that the inhibition is as robust in living mice as what we see in the tissue culture plate, although our hypothesis is supported by the observations that serum from $\mathrm{Cl}$-amidine-treated mice inhibited NET formation in control neutrophils and that deposition of NET-like material in the kidneys was decreased in the treated mice.

In the model described here, we cannot prove definitively that all outcomes downstream of PAD inhibition result from blockade of NET formation. It is reassuring that the mice tolerated the treatment well without notable adverse events, including no overt evidence of infection with normal flora. Total IgG levels (Figure 4D) as well as spleen weight and morphology (data not shown) were not affected by $\mathrm{Cl}$-amidine treatment. Also, while $\mathrm{Cl}$-amidine treatment robustly inhibited NET formation in vitro, it did not have any effect on $\mathrm{T}$ or $\mathrm{B}$ cell proliferation, apoptosis, or activation. Further, the work of others has shown that daily Cl-amidine treatment does not alter the frequency or absolute number of T cells (total and subsets), B cells, NK cells, or monocytes (32). Nevertheless, how PAD inhibition may directly affect other cells of the innate and adaptive immune systems relevant to SLE should be the focus of future investigation.

Interestingly we found that $\mathrm{Cl}$-amidine treatment resulted in higher circulating levels of both complement $\mathrm{C} 3$ and anti-dsDNA autoantibodies, while resulting in less deposition of both $\mathrm{C} 3$ and IgG in the kidneys. The $\mathrm{C} 3$ data are interesting in the context of a recent study demonstrating that NETs can activate the complement cascade, resulting in the consumption of $\mathrm{C} 3$ and $\mathrm{C} 4$, and in the recruitment of C1q, which protects against DNase-mediated degradation (14).

Another interesting result was the striking increase in antidsDNA levels with Cl-amidine treatment. While the traditional view in the clinical care of lupus patients is that anti-dsDNA levels rise with more active clinical disease (71), some studies have found the opposite - that, while increased levels may herald a flare, anti-dsDNA levels then fall as disease actually becomes active $(15,41)$; the hypothesis in these studies was that antidsDNA levels decrease as the result of accelerated tissue depo- 
sition during the flare. Indeed, we observed such a trend in our vehicle-treated mice, with anti-dsDNA levels rising through 20 weeks of age, but then falling as nephritis develops and immune complex deposition accelerates. This concept is also supported by the experimental work of others in that higher concentrations of extracellular DNA (especially larger DNA fragments) facilitate the clearance of anti-dsDNA from circulation $(42,43)$. There is even speculation that autoantibody levels may drop as a direct result of proteinuria (72). Therefore, we speculate that, by blocking NET formation, we are reducing the nidus of extracellular DNA available for immune complex formation and deposition. In support of this hypothesis is the statistically significant inverse correlation we observed between glomerular IgG deposition and serum anti-dsDNA levels (Supplemental Figure 7C) as well as the similar rise in anti-NETs and anti-CRAMP autoantibodies with Cl-amidine treatment (Supplemental Figure 6).

While Cl-amidine prevented immune complex deposition in the kidneys of NZM mice and decreased the presence of NET-like material in glomeruli, we were unable to demonstrate a statistically significant difference in either proteinuria or histologic scores. An important comment is that we only characterized histopathology at a single time point, given limitations imposed by the quantity of drug needed for daily treatment. It is possible that earlier or later time points would have detected significant differences in these parameters. Further, there is evidence to suggest that DNase may be downregulated in murine kidneys as nephritis develops (73), so even suppressed NET formation may be sufficient to cause damage in this nuclease-poor environment. Future studies examining histology at various time points will be needed to further dissect whether Cl-amidine can modulate renal damage in murine lupus or whether combinations of Cl-amidine and other drugs may be more effective than single drug treatments.

A particularly relevant therapeutic agent to consider, in the context of our data, is DNase. Given the clear association between lupus and anti-dsDNA antibodies as well as the recognition of lupus-like nephritis in DNase-deficient mice (74), recombinant DNase has long been considered as a possible treatment for lupus $(23,24,75)$. While initial murine studies suggested significant effectiveness against nephritis (24), a more thorough study, involving larger numbers of mice, was unable to replicate those findings, with only a modest and transient improvement in antidsDNA levels (23). Further, phase I studies in human patients with DNase have not been promising to date (75). In the context of our thrombosis model (which permitted for shorter-term therapy), recombinant DNase did replicate the phenotype of $\mathrm{Cl}$-amidine. And given the less than complete effectiveness of both $\mathrm{Cl}$-amidine and DNase against murine nephritis, one wonders whether combination therapy might prove more successful and this should be tested in future studies.

An important finding from our study is that PAD inhibition led to significant and robust improvements in vasculogenesis, endothelial function, and prothrombotic phenotype of lupusprone mice, consistent with the balance of studies to date pointing toward a toxic role for NETs at the endothelial surface (18, 21, $76)$. Indeed, aortic rings from $\mathrm{Cl}$-amidine-treated mice showed clear improvements in endothelium-dependent vasorelaxation, a marker of vascular health. This is particularly important in the context of SLE, where endothelial dysfunction is highly prevalent in lupus patients, a phenomenon that may predate atherosclerosis development $(77,78)$.
We have also previously shown that EPC function is impaired in $\operatorname{SLE}(28,45,79)$, and here Cl-amidine treatment markedly enhanced the function of bone marrow EPCs from NZM mice. In human patients, EPC dysfunction correlates with an enhanced type I IFN signature $(45,79)$, and we did find a trend toward abrogation of the IFN signature in the spleen compartment with $\mathrm{Cl}$-amidine treatment, although this data did not reach statistical significance. We therefore cannot definitively comment on whether NET-mediated activation of $\mathrm{pDCs}$, seen in ex vivo models of human SLE (16-18), appears in the NZM model. Future studies should consider whether Cl-amidine might more robustly suppress the type I IFN signature in other compartments (such as the bone marrow where the EPCs for this study originated) or at earlier time points before the mice begin to develop nephritis.

Finally, our results also support an important role for NETs in the hypercoagulable state of lupus patients and animal models of the disease $(28,51)$. Indeed, both DNase and Cl-amidine similarly prolonged time to carotid artery thrombosis induced by endothelial injury while significantly reducing the density of NETs in these thrombi. In this model, neutrophils undergoing NET formation were especially captured in the vicinity of the endothelium, which is interesting given the observation that endothelial activation can promote NET formation (76) as well as the recent evidence that NETs invade the endothelium and promote vascular damage in atherosclerosis $(53,80)$. We propose that the NET-dependent prothrombotic phenotype observed here has implications not only for the characteristic hypercoagulability of lupus patients (51), but also for patients with other inflammatory diseases characterized by enhanced NET formation, such ANCAassociated small vessel vasculitis, in which thrombosis is a wellrecognized complication of active disease $(54,81)$.

In summary, we have shown that NET formation is enhanced in at least 1 murine model of SLE. Our first attempt to modulate the impact of NET formation on lupus manifestations with the PAD inhibitor Cl-amidine - has significantly affected a number of features of the disease phenotype, while dramatically improving endothelial dysfunction and thrombosis risk. The next steps should focus on better understanding the impact of NET formation on vascular disease and development of autoimmune responses in other murine lupus models and in lupus patients. Indeed, our observations suggest that PAD inhibitors should be further explored as therapeutic tools in SLE and its complications.

\section{Methods}

Mice and drug treatment. Breeding pairs of lupus-prone NZM mice were a gift from Chaim Jacob (University of Southern California, Los Angeles, California, USA). Only female mice were used in the experiments described here. $\mathrm{BALB} / \mathrm{c}$ and C57BL/6 mice were purchased from The Jackson Laboratory. Mice were bred and housed in a specific pathogen-free barrier facility. Proteinuria was screened with Uristix-4 (Siemens), but definitive evaluation was with the Albuwell M Test kit and Creatinine Companion (Exocell).

$\mathrm{Cl}$-amidine was synthesized as has been described in detail previously (82). NZM mice were treated with either $\mathrm{Cl}$-amidine $(10 \mathrm{mg} / \mathrm{kg} / \mathrm{d})$ or an equal volume of PBS by daily subcutaneous injection, beginning at 12 weeks of age and through sacrifice at 26 weeks of age. Blood was collected by the tail-vein approach at least every 4 weeks after starting treatment with $\mathrm{Cl}$ amidine, and serum was stored at $-80^{\circ} \mathrm{C}$ for later batch processing. For in vitro experiments, $\mathrm{Cl}$-amidine was used at a concentration of $200 \mu \mathrm{M}$, including a 30-minute pretreatment in Locke's solution $(150 \mathrm{mM} \mathrm{NaCl}$, $5 \mathrm{mM} \mathrm{KCl}, 2 \mathrm{mM} \mathrm{CaCl}_{2}, 0.1 \%$ glucose, and $10 \mathrm{mM}$ HEPES buffer, $\mathrm{pH}$ 7.3). 
Neutrophil isolation. Peripheral blood neutrophils were obtained by first spinning heparinized blood on Histopaque-1083 (Sigma-Aldrich) according to the manufacturer's instructions, then subjecting the rbc layer to $1.5 \%$ dextran sedimentation. Remaining rbcs were lysed with RBC Lysis Buffer (multi-species) according to the manufacturer's instructions (eBioscience), and the remaining neutrophils were washed with PBS before use.

Bone marrow neutrophils were isolated as described previously (83). Briefly, bone marrow was flushed from femurs and tibias with HBSS with $15 \mathrm{mM}$ EDTA. Cells were then spun on a discontinuous Percoll gradient $(52 \%, 69 \%, 78 \%)$ at $1500 \mathrm{~g}$ for 30 minutes. Cells from the $69 \%-78 \%$ interface were isolated, and rbcs were lysed as above. Cells were more than $95 \%$ Ly6G-positive by flow cytometry and had the typical segmented nuclei of mature neutrophils by microscopy (data not shown).

NET quantification and microscopy. A protocol similar to what we have described previously was followed (18). Briefly, $1.5 \times 10^{5}$ neutrophils were seeded onto $22 \times 22 \mathrm{~mm}$ coverslips coated with $0.001 \%$ poly-L-lysine (Sigma-Aldrich). Neutrophils were then incubated in RPMI-1640 supplemented with L-glutamine, $2 \%$ BSA, and $10 \mathrm{mM}$ HEPES buffer. In experiments with either no stimulation or $2 \%$ mouse serum, incubation was for 4 hours at $37^{\circ} \mathrm{C}$. In experiments with $100 \mathrm{nM}$ PMA (Sigma-Aldrich) stimulation, incubation was for 12 hours. Cells were fixed with 4\% PFA and then blocked with $10 \%$ FBS; cells were not specifically permeabilized. DNA was stained with Hoechst 33342 (Invitrogen). Protein staining was with rabbit polyclonal antibodies to neutrophil elastase (ab21595; Abcam), MPO (A0398; Dako) or citrullinated histone H3 (ab5103; Abcam) and FITCconjugated anti-rabbit IgG (4052-02; SouthernBiotech). When 1\% mouse serum from experimental mice was used for staining, detection was with Texas red-conjugated anti-mouse IgG (T862; Invitrogen). After staining, cover slips were mounted in Prolong Gold Antifade Reagent (Invitrogen).

Images were collected with an Olympus microscope (IX70) and a CoolSNAP HQ2 monochrome camera (Photometrics) with Metamorph Premier software (Molecular Devices), typically at $\times 400$ magnification. Statistical background correction and image overlays were with Metamorph, and the recorded images were loaded onto Adobe Photoshop for further analysis, at which time NETs were manually quantified by 2 blinded observers. Decondensed nuclei (stained with Hoechst), which also stained positively for neutrophil elastase, were considered NETs and digitally recorded to prevent multiple counts. The percentage of NETs was calculated as the average of at least 5 fields, normalized to the total number of cells.

ELISA and multiplex assay. Commercial ELISAs for murine anti-dsDNA antibodies, total IgG, and C3 were purchased from Alpha Diagnostic and were performed according to the manufacturer's instructions. For inhouse ELISAs, recombinant CRAMP protein was purchased from AnaSpec. NET protein was prepared by inducing NZM bone marrow neutrophils with $100 \mathrm{nM}$ PMA for 12 hours, collecting supernatants, and then precipitating protein with $80 \%$ acetone. Protein was diluted to a concentration of $100 \mathrm{ng} / \mathrm{ml}$ with $0.05 \%$ Tween in PBS (wash buffer), and $100 \mu \mathrm{l}$ of the solution was used to coat high-binding EIA/RIA 96-well plates (Corning Inc.) overnight at $4{ }^{\circ} \mathrm{C}$. Plates were blocked with $10 \% \mathrm{FBS}$ in PBS and then incubated with experimental mouse serum diluted 1:100 in wash buffer. Secondary reagents were biotinylated anti-mouse IgG (B7264; Sigma-Aldrich) and HRP-conjugated streptavidin (7100-05; SouthernBiotech), both used at a 1:2000 dilution in wash buffer. A color change was elicited with $3,3^{\prime}, 5,5^{\prime}$-tetramethylbenzidine (TMB) substrate (Invitrogen), and the reaction was stopped with $0.16 \mathrm{M} \mathrm{H}_{2} \mathrm{SO}_{4}$. Between incubation steps, plates were washed 7 times with wash buffer. Final absorbance was measured at $450 \mathrm{~nm}$ with a Synergy HT Multi-Mode Microplate Reader (BioTek). A multiplex assay for 5 cytokines (IFN- $\gamma$, IL-2, IL-4, IL-5, and TNF- $\alpha$ ) was performed with a MILLIPLEX MAP Mouse Cytokine/Chemokine Magnetic Bead Panel (EMD Millipore) according to the manufacturer's instructions.
Western blotting. Proteins were resolved by $15 \%$ SDS-PAGE under denaturing conditions and then transferred to 0.45 -micron nitrocellulose membrane. Samples were probed with antibodies to MPO (A0398; Dako) and citrullinated histone $\mathrm{H} 3$ (ab5103; Abcam) or with $0.5 \%$ experimental mouse serum. Detection was with HRP-conjugated anti-rabbit IgG or antimouse IgG secondary antibodies (Jackson ImmunoResearch) and Western Lightning Plus-ECL (PerkinElmer).

Kidney harvesting and renal histopathology. Mice were anesthetized with pentobarbital, and kidneys were perfused with heparinized PBS (50 units/ml) at a rate of approximately $12 \mathrm{ml} / \mathrm{min}$ for 5 minutes via left-sided cardiac puncture. A portion of the cortex was fixed in Buffered Formalde-Fresh (Fisher Scientific), embedded in paraffin, and sectioned at 3-micron thickness for histopathology. At the same time, another cortical portion was frozen in Tissue-Tek OCT Compound (Sakura Finetek) and frozen at $-80^{\circ} \mathrm{C}$ until sectioning at 6 -micron thickness for immunofluorescence staining.

For histopathology, sections were stained by PAS and then scored in a blinded manner as described by our group previously (84). In brief, a semiquantitative scoring system $(0$, no involvement; 0.5 , minimal involvement of $<10 \%$; 1 mild involvement of $10 \%-30 \% ; 2$, moderate involvement of $31 \%-60 \%$; 3 , severe involvement of $>60 \%$ ) was used to assess 10 different parameters (mesangial hypercellularity, mesangial deposits, mesangial sclerosis, endocapillary cellular infiltrate, endocapillary sclerosis, endocapillary cellular crescents, endocapillary organized crescents, interstitial inflammation, tubular atrophy, and interstitial fibrosis). For glomerular indices, 30 glomeruli were examined and an average score was obtained. An activity and chronicity index was generated by compiling scores from groups of related parameters (for activity: mesangial hypercellularity, mesangial deposits, and endocapillary cellular infiltrate; for chronicity: endocapillary sclerosis, endocapillary organized crescents, tubular atrophy, and interstitial fibrosis).

Immunofluorescence staining was performed on frozen sections with Texas red-conjugated goat anti-mouse IgG (T862; Invitrogen) and FITCconjugated goat anti-mouse C3 (GC3-90F-Z; Immunology Consultants Laboratory). For both IgG and C3, glomerular staining was graded in blinded fashion by intensity on a 0 to $3+$ scale for at least 10 glomeruli per mouse; an average score was then calculated.

Assessment of endothelium-dependent vasorelaxation. Studies were performed as previously reported by our group (84). After euthanasia with pentobarbital and saline perfusion, thoracic aortas were excised, cleaned, and cut into 2-mm length rings. Endothelium was left intact, and aortic rings were mounted in a myograph system (Danish Myo Technology A/S). Vessels were bathed with warmed, aerated $\left(95 \% \mathrm{O}_{2} / 5 \% \mathrm{CO}_{2}\right)$ physiological salt solution (PSS). Aortic rings were set at $7 \mathrm{mN}$ passive tension and equilibrated for 1 hour, with washing every 20 minutes. Prior to performing drug-response curves, the vessels were contracted with PSS containing $100 \mathrm{mM}$ potassium chloride (KPSS) and then returned to PSS. Cumulative concentrations of phenylephrine (PE) $\left(10^{-9} \mathrm{M}\right.$ to $\left.10^{-6} \mathrm{M}\right)$ were then added to the bath to establish a concentration-response curve. A PE concentration corresponding to $80 \%$ maximum was added, and contraction was allowed to reach a stable plateau. To examine endothelium-dependent relaxation, Ach $\left(10^{-9} \mathrm{M}\right.$ to $\left.10^{-6} \mathrm{M}\right)$ was added cumulatively to the bath and a curve was generated. Finally, a normal vascular smooth muscle response was confirmed by washing out $\mathrm{PE}$ and Ach and then repeating the experiment with PE contraction and cumulative addition of sodium nitroprusside (SNP) $\left(10^{-9} \mathrm{~mol} / \mathrm{l}\right.$ to $\left.10^{-5} \mathrm{~mol} / \mathrm{l}\right)$ to the bath. Ach and SNP relaxation were expressed as percentage of $\mathrm{PE}$ contraction.

Quantification of $\mathrm{H}_{2} \mathrm{O}_{2}$ generation by neutrophils. The generation of $\mathrm{H}_{2} \mathrm{O}_{2}$ was quantified as previously described (85). In brief, $\mathrm{H}_{2} \mathrm{O}_{2}$ secretion from NZM bone marrow neutrophils stimulated with 100 nM PMA for 1 hour was assayed after pretreatment with either $\mathrm{Cl}$-amidine or $25 \mu \mathrm{M}$ diphenyl- 
eneiodonium (DPI) (Tocris Bioscience). $\mathrm{H}_{2} \mathrm{O}_{2}$ production was detected by a colorimetric assay, adding $50 \mu \mathrm{M}$ Amplex Red Reagent (Invitrogen) and $10 \mathrm{U} / \mathrm{ml}$ horseradish peroxidase (Sigma-Aldrich) to the culture medium. Absorbance was measured at $560 \mathrm{~nm}$, and linearity was assured with an $\mathrm{H}_{2} \mathrm{O}_{2}$ standard curve. The detection limit of the assay was $0.625 \mathrm{nM} \mathrm{H}_{2} \mathrm{O}_{2}$.

Lymphocyte activation and proliferation assays. Spleens were homogenized in PBS, and rbcs were lysed with a hypotonic salt solution. T and B cells were purified (Pan T Cell Isolation Kit and Pan B Cell Isolation Kit; Miltenyi Biotec) according to the manufacturer's instructions and were cultured in RPMI 1640 medium supplemented with $10 \%$ FBS. T cells were stimulated with anti-mouse CD3 $(5 \mu \mathrm{g} / \mathrm{ml}$; eBioscience) and anti-mouse CD28 $(5 \mu \mathrm{g} /$ $\mathrm{ml}$; eBioscience) for 48-72 hours in the presence or absence of $200 \mu \mathrm{M} \mathrm{Cl}$ amidine, while B cells were stimulated with LPS ( $1 \mu \mathrm{g} / \mathrm{ml}$; Sigma-Aldrich). $\mathrm{T}$ cell surface staining was with PE-conjugated anti-CD4, FITC-conjugated anti-CD25, or PE-Cy5-conjugated anti-CD69 (all from eBioscience). Proliferation was measured by the CellTrace CFSE Cell Proliferation Kit (Invitrogen) according to the manufacturer's instructions. Apoptosis was determined by staining with either FITC- or APC-conjugated anti-annexin V (BioLegend). Flow cytometry was on a BD FACSCalibur 3, and analysis was with FlowJo (Tree Star Inc.).

Quantitative real-time PCR. RNA isolation and real-time PCR for quantification of type I IFN-inducible genes were performed essentially as described previously (36). Briefly, RNA was isolated from spleens using TriPure Isolation Reagent (Roche) according to the manufacturer's instructions. cDNA was synthesized using MMLV RT (Invitrogen) and $1 \mu \mathrm{g}$ of RNA using a MyCycler Thermocyler (Bio-Rad). Five type I IFN-responsive genes and 1 housekeeping gene ( $\beta$-actin) were quantified by real-time PCR using SYBR Green PCR Master Mix (Applied Biosystems) according to the manufacturer's instructions. The primer sequences for the MX1, IRF7, IP-10, ISG15, and IFNG genes have been described by our group previously (36). Real-time PCR was carried out using an ABI PRISM 7900HT (Applied Biosystems). Transcripts were normalized using the $\beta$-actin gene, and $\Delta \mathrm{Ct}$ values (10 mice per group) were compared using a 2-tailed Student's $t$ test. Data are presented as relative fold change calculated by the formula $2^{\Delta \Delta \mathrm{Ct}}$.

Assessment of bone marrow EPC differentiation into mature endothelial cells. Bone marrow mononuclear cells were prepared by spinning on Histopaque 1083 (Sigma-Aldrich) according to the manufacturer's instructions and were then seeded into fibronectin-coated plates at a standardized density $\left(10^{6}\right.$ cells $\left./ \mathrm{cm}^{2}\right)$ in EGM-2 BulletKit medium (Lonza) with $5 \%$ heat-inactivated FBS. The medium was changed every 3 days, and on day 7, cells were incubated with fluorescein-labeled Griffonia Simplicifolia Lectin I (Vector) and DiI-labeled acetylated LDL (Biomedical Technologies) and then analyzed by fluorescence microscopy (Olympus IX70). Cells costaining with Lectin I and acetylated LDL were considered to be mature ECs and were quantified in 6 random $\times 100$ fields per well using CellC software, as described by us (28).

Induction of carotid artery thrombosis by photochemical injury. As described previously (28), mice were subjected to photochemical injury of the right carotid artery by rose bengal dye. Briefly, mice were anesthetized and placed under a dissecting microscope, at which point the right carotid artery was isolated. A Doppler flow probe (Transonic Systems) was applied, and rose bengal dye (Fisher Scientific) diluted in PBS was injected into the tail vein $(50 \mathrm{mg} / \mathrm{kg})$. A $1.5-\mathrm{mW}$ green light laser $(540 \mathrm{~nm}$; CVI Melles Griot) was applied to the desired site of injury from a distance of $6 \mathrm{~cm}$, and vessel flow was monitored until occlusive thrombosis occurred, defined as flow cessation for at least 10 minutes. DNase treatment was essentially as described previously $(47,52)$ using dornase alfa (Genentech); specifically, each mouse received 2 doses: $50 \mu \mathrm{g}$ by intraperitoneal injection 2 hours before rose bengal administration and $10 \mu \mathrm{g}$ by intravenous injection 10 minutes before rose bengal.

Thrombus immunostaining. Tissue was fixed with formalin (Buffered Formalde-Fresh; Sigma-Aldrich) and embedded in paraffin; 5-micron sections were then prepared. Alternatively, some thrombi were frozen in Tissue-Tek OCT Compound (Sakura Finetek) and frozen at $-80^{\circ} \mathrm{C}$ until sectioning at 10-micron thickness for immunofluorescence staining. For the immunohistochemistry experiments, deparaffinization and rehydration were with standard xylene-to-ethanol washes. Heat-induced epitope retrieval was achieved by boiling samples for 30 minutes in sodium citrate buffer (10 mM sodium citrate, $0.05 \%$ Tween $20, \mathrm{pH}$ 6.0). Samples were blocked in Tris-buffered saline with $1 \%$ BSA and either $10 \%$ FBS or $10 \%$ normal goat serum, depending on the secondary antibody. Primary antibodies were to citrullinated histone $\mathrm{H} 3$ (ab5103; Abcam) and CRAMP (PA-CRPL; Innovagen), while the secondary antibody was HRP-conjugated antirabbit Ig (NA9340V; Amersham Biosciences). Color change was detected with the DAB-Plus Substrate Kit (Invitrogen). For immunofluorescence experiments, the secondary antibody was FITC-conjugated anti-rabbit IgG (4052-02; SouthernBiotech). DNA was stained with Hoechst 33342 (Invitrogen). Publicly available ImageJ software (NIH) was used for area determination and quantification of each thrombus section, with at least 3 sections quantified and averaged per mouse. Volumes were determined by multiplying thrombus area by section depth.

Statistics. Unless otherwise indicated, results are presented as the mean \pm SEM. For most experiments, statistical analysis was performed using Student's $t$ test, and data were analyzed using GraphPad Prism software version 5. For the assessment of endothelial function, curves were first analyzed by asymmetric (5 parameter) logistic equation, and significance at individual data points was determined by 2-way ANOVA (GraphPad).

Study approval. All protocols were approved by the University of Michigan's Committee on Use and Care of Animals

\section{Acknowledgments}

We are grateful to Chaim Jacob for providing initial breeding pairs of NZM 2328 mice and to Chiao Guo for expert sectioning of carotid artery thrombi. Microscopy was performed in the Center for Live Cell Imaging (CLCI) at the University of Michigan. This work was supported by the NIH through PHS grant HL088419 (to M.J. Kaplan). The study was also funded in part by training grant T32 AR7080. J.S. Knight was supported by a Rheumatology Research Foundation Rheumatology Scientist Development Award. P.R. Thompson is supported by NIH grants GM079357 and CA151304.

Received for publication October 16, 2012, and accepted in revised form April 5, 2013.

Address correspondence to: Mariana J. Kaplan, Division of Rheumatology, Department of Internal Medicine, University of Michigan Medical School, 1150 W. Medical Center Drive; 5520 Medical Science Research Building-I, Ann Arbor, Michigan 48109-5680, USA. Phone: 734.936.7905; Fax: 734.763.4151; E-mail: makaplan@ umich.edu.
1. Roman MJ, et al. Prevalence and correlates of accelerated atherosclerosis in systemic lupus erythematosus. N Engl J Med. 2003;349(25):2399-2406.

2. Urowitz MB, Bookman AA, Koehler BE, Gordon

DA, Smythe HA, Ogryzlo MA. The bimodal mor- tality pattern of systemic lupus erythematosus. $A m$ JMed. 1976;60(2):221-225.

3. Tsokos GC. Systemic lupus erythematosus. NEngl JMed. 2011;365(22):2110-2121.

4. Kaplan MJ, Salmon JE. How does interferon-alpha insult the vasculature? Let me count the ways. Arthritis Rheum. 2011;63(2):334-336.

5. Elkon KB, Stone VV. Type I interferon and systemic lupus erythematosus. J Interferon Cytokine Res. 2011; 31(11):803-812. 
6. Crispin JC, Kyttaris VC, Terhorst C, Tsokos GC. T cells as therapeutic targets in SLE. Nat Rev Rheumatol. 2010;6(6):317-325.

7. Dorner T, Jacobi AM, Lee J, Lipsky PE. Abnormalities of $B$ cell subsets in patients with systemic lupus erythematosus. JImmunol Methods. 2011;363(2):187-197.

8. Knight JS, Kaplan MJ. Lupus neutrophils: 'NET' gain in understanding lupus pathogenesis. Curr Opin Rheumatol. 2012;24(5):441-450.

9. Kobayashi SD, DeLeo FR. Role of neutrophils in innate immunity: a systems biology-level approach. Wiley Interdiscip Rev Syst Biol Med. 2009;1(3):309-333.

10. Brinkmann V, et al. Neutrophil extracellular traps kill bacteria. Science. 2004;303(5663):1532-1535.

11. Kaplan MJ, Radic M. Neutrophil extracellular traps: double-edged swords of innate immunity. J Immunol. 2012;189(6):2689-2695.

12. Brinkmann V, Zychlinsky A. Neutrophil extracellular traps: Is immunity the second function of chromatin? J Cell Biol. 2012;198(5):773-783

13. Hakkim A, et al. Impairment of neutrophil extracellular trap degradation is associated with lupus nephritis. Proc Natl Acad Sci U S A. 2010; 107(21):9813-9818.

14. Leffler J, et al. Neutrophil extracellular traps that are not degraded in systemic lupus erythematosus activate complement exacerbating the disease. J Immunol. 2012;188(7):3522-3531.

15. Tan EM, Schur PH, Carr RI, Kunkel HG. Deoxybonucleic acid (DNA) and antibodies to DNA in the serum of patients with systemic lupus erythematosus. J Clin Invest. 1966;45(11):1732-1740.

16. Lande R, et al. Neutrophils activate plasmacytoid dendritic cells by releasing self-DNA-peptide complexes in systemic lupus erythematosus. Sci Transl Med. 2011;3(73):73ra19.

17. Garcia-Romo GS, et al. Netting neutrophils are major inducers of type I IFN production in pediatric systemic lupus erythematosus. Sci Transl Med. 2011;3(73):73ra20.

18. Villanueva E, et al. Netting neutrophils induce endothelial damage, infiltrate tissues, and expose immunostimulatory molecules in systemic lupus erythematosus. J Immunol. 2011;187(1):538-552.

19. Liu CL, et al. Specific post-translational histone modifications of neutrophil extracellular traps as immunogens and potential targets of lupus autoantibodies. Arthritis Res Ther. 2012;14(1):R25.

20. Thomas GM, et al. Extracellular DNA traps are associated with the pathogenesis of TRALI in humans and mice. Blood. 2012;119(26):6335-6343.

21. Caudrillier A, et al. Platelets induce neutrophil extracellular traps in transfusion-related acute lung injury. J Clin Invest. 2012;122(7):2661-2671.

22. Meng W, et al. Depletion of neutrophil extracellular traps in vivo results in hypersusceptibility to polymicrobial sepsis in mice. Crit Care. 2012;16(4):R137.

23. Verthelyi D, Dybdal N, Elias KA, Klinman DM DNAse treatment does not improve the survival of lupus prone (NZB x NZW)F1 mice. Lupus. 1998; $7(4): 223-230$.

24. Macanovic M, Sinicropi D, Shak S, Baughman S, Thiru S, Lachmann PJ. The treatment of systemic lupus erythematosus (SLE) in NZB/W F1 hybrid mice; studies with recombinant murine DNase and with dexamethasone. Clin Exp Immunol. 1996; 106(2):243-252.

25. Li P, Li M, Lindberg MR, Kennett MJ, Xiong N, Wang Y. PAD4 is essential for antibacterial innate immunity mediated by neutrophil extracellular traps. J Exp Med. 2010;207(9):1853-1862.

26. Hemmers S, Teijaro JR, Arandjelovic S, Mowen KA. PAD4-mediated neutrophil extracellular trap formation is not required for immunity against influenza infection. PLoS One. 2011;6(7):e22043.

27. Ermert D, Urban CF, Laube B, Goosmann C, Zychlinsky A, Brinkmann V. Mouse neutrophil extracellular traps in microbial infections. J Innate
Immun. 2009;1(3):181-193.

28. Thacker SG, et al. Type I interferons modulate vascular function, repair, thrombosis, and plaque progression in murine models of lupus and atherosclerosis. Arthritis Rheum. 2012;64(9):2975-2985.

29. Agrawal H, et al. Deficiency of type I IFN receptor in lupus-prone New Zealand mixed 2328 mice decreases dendritic cell numbers and activation and protects from disease. J Immunol. 2009;183(9):6021-6029.

30. Luo Y, et al. Inhibitors and inactivators of protein arginine deiminase 4 : functional and structural characterization. Biochemistry. 2006:45(39):11727-11736.

31. Chumanevich AA, et al. Suppression of colitis in mice by Cl-amidine: a novel peptidylarginine deiminase inhibitor. Am J Physiol Gastrointest Liver Physiol. 2011;300(6):G929-G938.

32. Willis VC, et al. N-alpha-benzoyl-N5-(2-chloro1 -iminoethyl)-L-ornithine amide, a protein arginine deiminase inhibitor, reduces the severity of murine collagen-induced arthritis. J Immunol. 2011; 186(7):4396-4404.

33. Chavanas $\mathrm{S}$, et al. Comparative analysis of the mouse and human peptidylarginine deiminase gene clusters reveals highly conserved non-coding segments and a new human gene, PADI6. Gene. 2004;330:19-27.

34. Kessenbrock K, et al. Netting neutrophils in autoimmune small-vessel vasculitis. Nat Med. 2009; 15(6):623-625.

35. Sangaletti S, et al. Neutrophil extracellular traps mediate transfer of cytoplasmic neutrophil antigens to myeloid dendritic cells towards ANCA induction and associated autoimmunity. Blood. 2012; 120(15):3007-3018

36. Thacker SG, Duquaine D, Park J, Kaplan MJ. Lupus-prone New Zealand Black/New Zealand White F1 mice display endothelial dysfunction and abnormal phenotype and function of endothelial progenitor cells. Lupus. 2010;19(3):288-299.

37. Luo Y, Knuckley B, Lee YH, Stallcup MR, Thompson PR. A fluoroacetamidine-based inactivator of protein arginine deiminase 4: design, synthesis, and in vitro and in vivo evaluation. J Am Chem Soc. 2006; 128(4):1092-1093.

38. Bao L, Quigg RJ. Complement in lupus nephritis: the good, the bad, and the unknown. Semin Nephrol. 2007;27(1):69-80

39. Schur PH, Sandson J. Immunologic factors and clinical activity in systemic lupus erythematosus. NEngl J Med. 1968;278(10):533-538.

40. Waters ST, et al. NZM2328: a new mouse model of systemic lupus erythematosus with unique genetic susceptibility loci. Clin Immunol. 2001; 100(3):372-383.

41. Ho A, Magder LS, Barr SG, Petri M. Decreases in anti-double-stranded DNA levels are associated with concurrent flares in patients with systemic lupus erythematosus. Arthritis Rheum. 2001;44(10):2342-2349.

42. Horgan C, Burge J, Crawford L, Taylor RP. The kinetics of $[3 \mathrm{H}]$-dsDNA/anti-DNA immune complex formation, binding by red blood cells, and release into serum: effect of DNA molecular weight and conditions of antibody excess. I Immunol. 1984;133(4):2079-2084

43. Jones FS, Pisetsky DS, Kurlander RJ. The clearance of a monoclonal anti-DNA antibody following administration of DNA in normal and autoimmune mice. Clin Immunol Immunopathol. 1986;39(1):49-60.

44. Denny MF, et al. A distinct subset of proinflammatory neutrophils isolated from patients with systemic lupus erythematosus induces vascular damage and synthesizes type I IFNs. J Immunol. 2010; 184(6):3284-3297.

45. Somers EC, et al. Type I interferons are associated with subclinical markers of cardiovascular disease in a cohort of systemic lupus erythematosus patients. PLoS One. 2012;7(5):e37000.

46. Fuchs TA, et al. Extracellular DNA traps promote thrombosis. Proc Natl Acad Sci U S A. 2010;
107(36):15880-15885

47. Brill A, et al. Neutrophil extracellular traps promote deep vein thrombosis in mice. J Thromb Haemost. 2012;10(1):136-144.

48. van Montfoort ML, et al. Circulating nucleosomes and neutrophil activation as risk factors for deep vein thrombosis. Arterioscler Thromb Vasc Biol. 2013; 33(1):147-151.

49. Massberg S, et al. Reciprocal coupling of coagulation and innate immunity via neutrophil serine proteases. Nat Med. 2010;16(8):887-896.

50. von Bruhl ML, et al. Monocytes, neutrophils, and platelets cooperate to initiate and propagate venous thrombosis in mice in vivo. J Exp Med. 2012; 209(4):819-835

51. Tektonidou MG, Laskari K, Panagiotakos DB, Moutsopoulos HM. Risk factors for thrombosis and primary thrombosis prevention in patients with systemic lupus erythematosus with or without antiphospholipid antibodies. Arthritis Rheum. 2009;61(1):29-36.

52. Demers $\mathrm{M}$, et al. Cancers predispose neutrophils to release extracellular DNA traps that contribute to cancer-associated thrombosis. Proc Natl Acad SciUS A. 2012;109(32):13076-13081.

53. Doring Y, et al. Lack of neutrophil-derived CRAMP reduces atherosclerosis in mice. Circ Res. 2012; 110(8):1052-1056.

54. Nakazawa D, Tomaru U, Yamamoto C, Jodo S, Ishizu A. Abundant neutrophil extracellular traps in thrombus of patient with microscopic polyangiitis. Front Immunol. 2012;3:333.

55. Kaplan MJ. Neutrophils in the pathogenesis and manifestations of SLE. Nat Rev Rheumatol. 2011; 7(12):691-699.

56. Cochrane CG, Unanue ER, Dixon FJ. A role of polymorphonuclear leukocytes and complement in nephrotoxic nephritis. J Exp Med. 1965;122:99-116.

57. Rosetti $F$, et al. Human lupus serum induces neutrophil-mediated organ damage in mice that is enabled by Mac-1 deficiency. J Immunol. 2012; 189(7):3714-3723.

58. Austin HA. Diffuse proliferative lupus nephritis: identification of specific pathologic features affecting renal outcome. Kidney Int. 1984;25(4):689-695.

59 . Hotta $\mathrm{O}$, et al. Role of neutrophil elastase in the development of renal necrotizing vasculitis. Clin Nephrol. 1996;45(4):211-216

60. Henson PM. Pathologic mechanisms in neutrophilmediated injury. Am J Pathol. 1972;68(3):593-612.

61. Johnson RJ, Couser WG, Alpers CE, Vissers M, Schulze M, Klebanoff SJ. The human neutrophil serine proteinases, elastase and cathepsin G, can mediate glomerular injury in vivo. J Exp Med. 1988; 168(3):1169-1174.

62. Sthoeger ZM, Bezalel S, Chapnik N, Asher I, Froy O. High alpha-defensin levels in patients with systemic lupus erythematosus. Immunology. 2009; 127(1):116-122.

63. Vordenbaumen S, et al. Elevated levels of human beta-defensin 2 and human neutrophil peptides in systemic lupus erythematosus. Lupus. 2010; 19(14):1648-1653.

64. Ma CY, et al. Elevated plasma level of HMGB1 is associated with disease activity and combined alterations with IFN-alpha and TNF-alpha in systemic lupus erythematosus. Rheumatol Int. 2012; 32(2):395-402.

65. Mathian A, Weinberg A, Gallegos M, Banchereau J, Koutouzov S. IFN-alpha induces early lethal lupus in preautoimmune (New Zealand Black x New Zealand White) F1 but not in BALB/c mice.J Immunol. 2005;174(5):2499-2506.

66. Hron JD, Peng SL. Type I IFN protects against murine lupus. J Immunol. 2004;173(3):2134-2142.

67. Parker H, Dragunow M, Hampton MB, Kettle AJ, Winterbourn CC. Requirements for NADPH oxidase and myeloperoxidase in neutrophil extracellular 
trap formation differ depending on the stimulus. J Leukoc Biol. 2012;92(4):841-849.

68. Campbell AM, Kashgarian M, Shlomchik MJ. NADPH oxidase inhibits the pathogenesis of systemic lupus erythematosus. Sci Transl Med. 2012; 4(157):157ra141.

69. Rohrbach AS, Slade DJ, Thompson PR, Mowen KA. Activation of PAD4 in NET formation. Front Immunol. 2012;3:360.

70. Guiducci C, et al. Autoimmune skin inflammation is dependent on plasmacytoid dendritic cell activation by nucleic acids via TLR7 and TLR9. J Exp Med. 2010;207(13):2931-2942.

71. Isenberg DA, Manson JJ, Ehrenstein MR, Rahman A. Fifty years of anti-ds DNA antibodies: are we approaching journey's end? Rheumatology (Oxford). 2007;46(7):1052-1056.

72. Macanovic M, Hogarth MB, Lachmann PJ. Anti-DNA antibodies in the urine of lupus nephritis patients. Nephrol Dial Transplant. 1999;14(6):1418-1424.

73. Fismen S, Mortensen ES, Rekvig OP. Nuclease deficiencies promote end-stage lupus nephritis but not nephritogenic autoimmunity in (NZB x NZW) F1 mice. Immunol Cell Biol. 2011;89(1):90-99.

74. Jacob M, Napirei M, Ricken A, Dixkens C, Mannherz HG. Histopathology of lupus-like nephritis in Dnase1-deficient mice in comparison to NZB/W F1 mice. Lupus. 2002;11(8):514-527.

75. Davis JC, et al. Recombinant human Dnase I (rhDNase) in patients with lupus nephritis. Lupus. 1999;8(1):68-76.

76. Gupta AK, et al. Activated endothelial cells induce neutrophil extracellular traps and are susceptible to NETosis-mediated cell death. FEBS Lett. 2010; 584(14):3193-3197.

77. Kahlenberg JM, Kaplan MJ. The interplay of inflammation and cardiovascular disease in systemic lupus erythematosus. Arthritis Res Ther. 2011;13(1):203.

78. Westerweel PE, Verhaar MC. Endothelial progenitor cell dysfunction in rheumatic disease. Nat Rev Rheumatol. 2009;5(6):332-340.

79. Denny MF, et al. Interferon-alpha promotes abnormal vasculogenesis in lupus: a potential pathway for premature atherosclerosis. Blood. 2007; 110(8):2907-2915.

80. Megens RT, et al. Presence of luminal neutrophil extracellular traps in atherosclerosis. Thromb Haemost. 2012;107(3):597-598.

81. Tichelaar YI, Kluin-Nelemans HJ, Meijer K. Infections and inflammatory diseases as risk factors for venous thrombosis. A systematic review. Thromb Haemost. 2012;107(5):827-837.

82. Causey CP, Thompson PR. An improved synthesis of haloaceteamidine-based inactivators of protein arginine deiminase 4 (PAD4). Tetrahedron Lett. 2008;49(28):4383-4385.

83. Boxio R, Bossenmeyer-Pourie C, Steinckwich N, Dournon C, Nusse O. Mouse bone marrow contains large numbers of functionally competent neutrophils. J Lenkoc Biol. 2004;75(4):604-611.

84. Zhao W, et al. The peroxisome proliferator-activated receptor gamma agonist pioglitazone improves cardiometabolic risk and renal inflammation in murine lupus. J Immunol. 2009;183(4):2729-2740.

85. Serezani CH, Aronoff DM, Jancar S, Mancuso P, Peters-Golden M. Leukotrienes enhance the bactericidal activity of alveolar macrophages against Klebsiella pneumoniae through the activation of NADPH oxidase. Blood. 2005;106(3):1067-1075. 